\title{
The Different Roles of Hedonic and Eudaimonic Motives for Activities in Predicting Functioning and Well-Being Experiences
}

\author{
Laura Giuntoli $^{1}$ (D) Federica Condini ${ }^{1} \cdot$ Francesco Ceccarini $^{1} \cdot$ Veronika Huta $^{2}$. \\ Giulio Vidotto ${ }^{1}$
}

Published online: 16 July 2020

(C) The Author(s) 2020

\begin{abstract}
Research on hedonic and eudaimonic orientations has previously focused on their effects on well-being experiences. Very little is known about their associations with functioning. A preliminary objective of the study was to establish the factorial validity of the Hedonic and Eudaimonic Motives for Activities-Revised (HEMA-R) on an Italian sample $(\mathrm{N}=461)$ through a confirmatory factor analysis. The main objective was to study the distinctive patterns of correlates between different types of orientations to well-being and several outcome measures of positive experiences (flourishing, life satisfaction, positive affect), negative experiences (negative affect, depression, anxiety, stress) and functioning (dispositional coping strategies) by means of a multivariate linear model. The Italian version of the HEMA-R showed a three-factor structure, namely eudaimonic, pleasure, and relaxation orientations. Pleasure orientation was positively related to positive experiences of wellbeing and negatively related to negative experiences. Eudaimonic orientation was associated with positive experiences. Furthermore, eudaimonic orientation showed a positive relation with several adaptive coping strategies, whereas relaxation was associated with avoidant coping strategies. The results reflect that pleasure orientation is aimed at achieving pleasant feelings, while Eudaimonic orientation is aimed at living well. Our findings also suggest that pleasure orientation may reflect the "pursuit of pleasure" component of Hedonia, while relaxation orientation may reflect its "pain avoidance" component. Overall, this study supports the importance of distinguishing between Eudaimonia, and the pleasure and relaxation components of Hedonia to predict individual differences in subjective experiences and functioning.
\end{abstract}

Keywords HEMA-R $\cdot$ Hedonia $\cdot$ Eudaimonia $\cdot$ Hedonic orientations $\cdot$ Eudaimonic orientations $\cdot$ Coping strategies

Electronic supplementary material The online version of this article (https://doi.org/10.1007/s1090 2-020-00290-0) contains supplementary material, which is available to authorized users.

Laura Giuntoli

laura.giuntoli@phd.unipd.it

1 Department of General Psychology, University of Padova, Via Venezia 8, 35131 Padova, PD, Italy

2 School of Psychology, University of Ottawa, Ottawa, Canada 


\section{Introduction}

An essential question investigated by philosophers, psychologists and researchers of human sciences concerns how well-being can be achieved. There have historically been two main perspectives on the definition of well-being, namely Hedonia and Eudaimonia. According to the hedonic viewpoint, the key to well-being is the pursuit of physical and emotional pleasure, enjoyment and comfort, whereas the eudaimonic view suggests that well-being can be fulfilled by seeking to use and develop the best in oneself, consistently with one's own values (Huta and Ryan 2010).

Assuming that individuals can be mainly hedonically and/or eudaimonically oriented, an important research question that we want to address is if individuals can manifest different types of psychological outcomes depending on their orientations to well-being.

The term orientations to well-being was coined by Peterson et al. (2005) and later used in the literature review by Huta and Waterman (2014) to specifically denote the class of definitions that include values, ideals, personal reasons, motives, priorities, and goals that define the intentions underlying behaviors.

According to Huta (2016), the contents of orientations can be distinguished as either hedonic or eudaimonic. When the focus relies on the process underlying the pursuit of happiness, there are evidences of individual differences in choices of personal goals (Deci and Ryan 2000; Kasser and Ryan 2001) and in preferences of which existential needs individuals are aiming to satisfy (Peterson et al. 2005). Even if two persons achieve the same level of well-being (i.e., at the level of the outcome measures), the motivations underlying their life paths could be very different. For example, one may achieve happiness by satisfying her or his own need for a pleasant life (i.e., hedonic orientation to well-being), while another person may feel happy because of her or his motivation to engage in prosocial activities (i.e., eudaimonic orientation to well-being).

A preliminary topic to address for our investigation on the hedonic and eudaimonic orientations to well-being concerns the choice of the measurement instrument and its factorial properties. In the present study, we employed a revised version of the Hedonic and Eudaimonic Motives for Activities (HEMA), namely the HEMA-R. The HEMA is a nine-items scale developed by Huta and Ryan (2010) to separate the constitutive elements of Eudaimonia (authenticity, meaning, excellence and growth) and Hedonia (pleasure and comfort). The HEMA-R authors (Huta and Waterman 2014), with respect to the HEMA, expanded the eudaimonic orientation subscale by including an item concerning the concept of meaning, given that the evidence outlined an important element of Eudaimonia involves caring about and contributing to something larger than themselves (Huta 2016; Seligman 2002). Although several studies confirmed a two-factor solution for the HEMA (e.g., Anić 2014; Huta and Ryan 2010), other studies showed that a better model fit was provided by a threefactor solution obtained by decomposing the hedonic orientation in pleasure and relaxation (Asano et al. 2018; Bujacz et al. 2014). Because of the mixed results on the factorial structure of the instrument, and because we employed an adaptation of the HEMA-R in a new linguistic and cultural context, we deemed testing its factorial validity in our sample as important.

Therefore, a preliminary objective of our study was to establish the factorial validity of the HEMA-R in the Italian context. First, we determined if a best factorial solution would be provided by a two-dimensional or a three-dimensional model of the HEMA-R. Subsequently, we tested the validity of the HEMA-R subscales in predicting several well-being and ill-being outcomes, as well as coping strategies. 
While orientations and behaviors represent ways of living, experiences and functioning generally represents the outcomes of the previous ones (Huta and Waterman 2014). Indeed, orientations play a key role in predicting different types of well-being outcomes given that they represent the underlying reasons for individual choices and behaviors.

The aim of the present study was to explore which typical experiences and functioning styles are specifically associated with hedonic and eudaimonic orientations to well-being.

According to Huta and Waterman (2014), within the experiences category are included both momentary and typical emotional states experienced by individuals (i.e., a predominance of positive affect over negative affect), as well as their cognitive evaluations on how well they feel (e.g., life satisfaction, flourishing). To provide a complete picture of the experiences category, it is also important to consider the negative pole of the mental health continuum by means of individual experiences of depression, anxiety, and stress (see Keyes 2002 for a discussion on the concept of full mental health). Finally, functioning can be defined as the qualitative connotation of behaviors and thinking, in that it underlines the adaptive (or maladaptive) self-regulation of individuals.

Several researchers have explored the relationship between measures of well-being orientations and experiences, suggesting that hedonic and eudaimonic motives for action can be distinguished on the basis of their different types of outcomes. For instance, Henderson et al. (2013) showed that, on the one hand, hedonic motives for activities fostered positive affect, carefreeness, vitality, and life satisfaction, whereas they reduced negative affect, depression, and stress. On the other hand, eudaimonic orientations predicted meaning in life and elevating experience. Notably, both hedonic and eudaimonic motives predicted flourishing.

However, less attention has been directed to the study of the relationship between different types of orientations to well-being and indicators of individual functioning. Coping strategies may be considered a useful proxy for functioning because they encompass both adaptive and maladaptive response styles in face of life situations. Although coping strategies were mainly studied in the context of trauma and adversities, some studies highlighted a strong association between coping strategies and well-being in the general population. For instance, in a four-year longitudinal study Moulin et al. (2017) explored a wide range of variables as potential predictors of excellent well-being measured by the Mental Health Continuum-Short Form (MHC-SF; Keyes 2002, 2005). The authors found that the strongest effects on well-being were those related to the ability to cope with stress. Furthermore, a perspective study on adolescents outlined that purpose in life predicted higher levels of well-being outcomes after one year. The relationship between purpose in life and well-being was mediated by both higher adaptive and lower maladaptive coping strategies. However, to our knowledge the specific relationship between hedonic and eudaimonic orientations to well-being and coping strategies was never directly investigated, though it can be speculated that they should be quite related to each other. For example, drawing from the study by Chua et al. (2015), purpose in life could be considered a eudaimonic motivation that generates a goal oriented behavior and, in turn, a proactive style of coping. By contrast, a lack of future orientation may lead individuals to seek immediate pleasure and avoid pain (i.e., hedonic orientation) by carrying on avoidant coping strategies when they face difficulties in life. Furthermore, in a recent study, Ortner et al. (2018) investigated the relationship between hedonic and eudaimonic motives on the one side, and adaptive and dysfunctional response styles in emotional regulation on the other. Results showed that the eudaimonic motives, over the hedonic ones, were the best predictors of well-being because of their stronger associations with adaptive choices in emotional regulation. On the basis of these findings, it is worth further development to determine which specific adaptive or 
dysfunctional response strategies are associated with hedonic and eudaimonic motives for action. By examining dispositional coping strategies, we may clarify which forms of functioning can be predicted by specific individual orientations.

The main objective of the present research was to explore to what extent different types of orientations to well-being were differentially associated to several outcomes of (a) positive experiences, (b) negative experiences, and (c) functioning: understood as functional or dysfunctional coping strategies.

Our predictions on the aforementioned associations are as follows.

The relationship between motives for activities and positive experiences. Because previous research (Henderson et al. 2013; Huta and Ryan 2010) showed that a hedonic orientation is more associated than a eudaimonic orientation with positive affect, we expected hedonic orientation to show a stronger relationship with measures of positive experiences over eudaimonic orientation.

The relationship between motives for activities and negative experiences. With reference to the study by Henderson et al. (2013), it should be expected that depression, anxiety, and stress would be more strongly and negatively associated with hedonic orientation than with eudaimonic orientation. In particular, given that depression is characterized by anhedonia, it could be considered the opposite pole of hedonic orientation. As a consequence, seeking pleasure and depression should show the strongest negative association compared to the other dimensions of orientations and negative experiences.

The relationship between motives for activities and functioning. We expect a prevalence of adaptive coping strategies associated to eudaimonic orientation over the hedonic orientation. For example, because a eudaimonic motive for action would be to use the best in oneself and pursue excellence or a personal ideal, we expect a positive relationship with approach coping styles (i.e., active coping, planning, and suppression of competing activities). Also, we expect a positive relationship between eudaimonic orientation and positive reinterpretation and growth because they are central aspects of Eudaimonia (Ryff 2014).

Seeking pleasure and avoiding pain are the essential characteristics of the "hedonictype" person. Although this represents a simplistic representation of a real attitude of an individual, we predict that a predominance for hedonic orientation may be principally associated to maladaptive coping strategies. Because of the propensity to avoid pain, individuals with a hedonic orientation may use avoidant coping (e.g., denial, behavioral, and mental disengagement, and substance use) when they face difficulties in life. Nevertheless, we expect that hedonic orientation may also be associated with more adaptive coping strategies, such as humor.

\section{Method}

\subsection{Participants}

We enrolled participants by means of adverts posted on Italian websites and social network groups. The study was conducted in accordance with the Declaration of Helsinki. The respondents were informed about the volunteer and confidential nature of the study, and they provided their electronic informed consents prior to completing the online questionnaires. The sample was comprised of 461 young adults ( $72 \%$ females) aged between 18 
and 30 years old $(\mathrm{M}=24.25 ; \mathrm{SD}=2.96)$. Of the participants, $69 \%$ were students, $25 \%$ were employed, and $6 \%$ were unemployed. For education, $91 \%$ of the employed and $86 \%$ of the unemployed had at least a bachelor's degree. Most of the participants (84\%) were not married or cohabiting, and only seven participants had children.

\subsection{Procedure}

The HEMA-R was adapted for Italy using a translation/back-translation process. Two independent translators translated the original English version of the HEMA-R into Italian. The first author compared the two translations and reconciled minor discrepancies. The Italian version of the HEMA-R was then translated back to English independently by a native English speaker who was blinded to the original questionnaire. The back translation to English was then compared with the original English version, confirming the adequacy of the Italian version of the HEMA-R.

\subsection{Measures}

Hedonic and Eudaimonic Motives for Activities-Revised (HEMA-R; Huta and Waterman, 2014). The HEMA-R is a 10-item self-report measure of hedonic and eudaimonic orientations. Respondents rate on a 7-point Likert-type scale (from $1=$ "not at all" to $7=$ "very much") to what degree they typically approach their activities with eudaimonic (e.g., "Seeking to use the best in yourself") or hedonic (e.g., "Seeking enjoyment", "Seeking relaxation") intentions.

Scale of Positive and Negative Experience (SPANE; Diener et al. 2010; Giuntoli et al. 2017). Positive and negative affect were assessed using the two 6-item subscales of the SPANE. Participants were asked to rate the frequency with which they experienced the feelings indicated by each item over the past four weeks by means of a 5-point Likert-type scale (from $1=$ "very rarely or never", to 5 = "very often or always").

Satisfaction With Life Scale (SWLS; Diener et al. 1985). Life satisfaction was measured by the five items of the SWLS. Participants responded to each item by indicating on a 7-point Likert-type scale ranging from 1 (strongly disagree) to 7 (strongly agree) their satisfaction with life as a whole. According to Huta (2016), the SWLS, through its general wording, can be conceived as an outcome measure that may encompass both hedonic and eudaimonic contents.

Flourishing Scale (FS; Diener et al. 2010; Giuntoli et al. 2017). The FS is an 8-item scale assessing positive psychological functioning. Typical items include: "I lead a purposeful and meaningful life", "My social relationships are supportive and rewarding" and "I am engaged and interested in my daily activities". Responses are scored on a 7-point Likert-type scale.

Depression Anxiety Stress Scale 21 (DASS-21; Bottesi et al. 2015; Lovibond and Lovibond 1995). The DASS-21 consists of three 7-item subscales measuring depression (e.g., hopelessness, anHedonia), anxiety (e.g., autonomic arousal), and stress (e.g., difficulty relaxing). Responses are scored on a 4-point Likert-type scale assessing the frequency of symptoms, ranging from 1 (never) to 4 (always).

Coping Orientation to Problems Experienced (COPE; Carver et al. 1989). The COPE is composed of a total of 60 items rated on a 4-point Likert-type scale ranging from "I never do this" to "I do this very often". Coping strategies are assessed by 15 subscales. The items are summed for each subscale separately, with higher scores indicating a 
greater use of the coping strategy in question. In the present study, we used a dispositional version of the COPE adapted and translated into Italian by Sica et al. (1997, 2008). Carver et al. (1989) defined the following subscales to assess coping strategies: active coping (e.g., "I take direct action to get around the problem"); planning (e.g., "I make a plan of action"); suppression of competing activities (e.g., "I put aside other activities in order to concentrate on this"); restraint (e.g., "I force myself to wait for the right time to do something"); use of instrumental social support (e.g., "I try to get advice from someone about what to do"); use of emotional social support (e.g., "I try to get emotional support from friends or relatives"); focus on and venting of emotions (e.g., "I let my feelings out"); positive reinterpretation and growth (e.g., "I try to grow as a person as a result of the experience"); behavioral disengagement (e.g., "I just give up trying to reach my goal"); humor (e.g., "I kid around about it"); acceptance (e.g., "I learn to live with it"); denial (e.g., "I pretend that it hasn't really happened"); mental disengagement (e.g., "I go to movies or watch TV, to think about it less"); substance use (e.g., "I use alcohol or drugs to make myself feel better"); religious coping (e.g., "I seek God's help").

\subsection{Data Analysis}

All the analyses were conducted with the software R ( R Core Team 2018). The factorial structure of the HEMA-R was evaluated by means of a confirmatory factor analysis (CFA) via the R package lavaan (Rosseel 2012). Given the data's ordinal nature, we performed the CFA with the diagonal weighted least squares (DWLS) estimator. To determine the goodness of the fit of the model, we considered the comparative fit index (CFI), the Tucker-Lewis index (TLI), the root-mean-square error of approximation (RMSEA), and the standardized root mean square residual (SRMR). The thresholds considered as indicative of a good fit are values greater than or equal to 0.95 for the TLI and CFI, less than or equal to 0.06 for RMSEA, and less than or equal to 0.08 for SRMR (Hu and Bentler 1998).

Next, we evaluated the predictive power of the HEMA-R by means of a multivariate linear model in which the HEMA-R subscales acted as explanatory variables, while the outcome measures of positive experiences (flourishing, life satisfaction, positive affect), negative experiences (negative affect, depression, anxiety, stress), and functioning (dispositional coping strategies) were set as response variables. The total explained variance of the model was computed by the total determination coefficient (TDC; Pastore 2012).

\section{Results}

\subsection{Preliminary Analyses}

Table 1 shows the descriptive statistics of the HEMA-R's items, as well as the original English wording and the Italian translations. Scores on items were skewed positively, except for item 7 ("seeking to take it easy") that was centered on the value of 3.56. The highest mean values were those for the items 3 and 8, meaning that most of the people in our sample tended to manifest a eudaimonic orientation to activities by striving to do their best and behaving according to their values. 


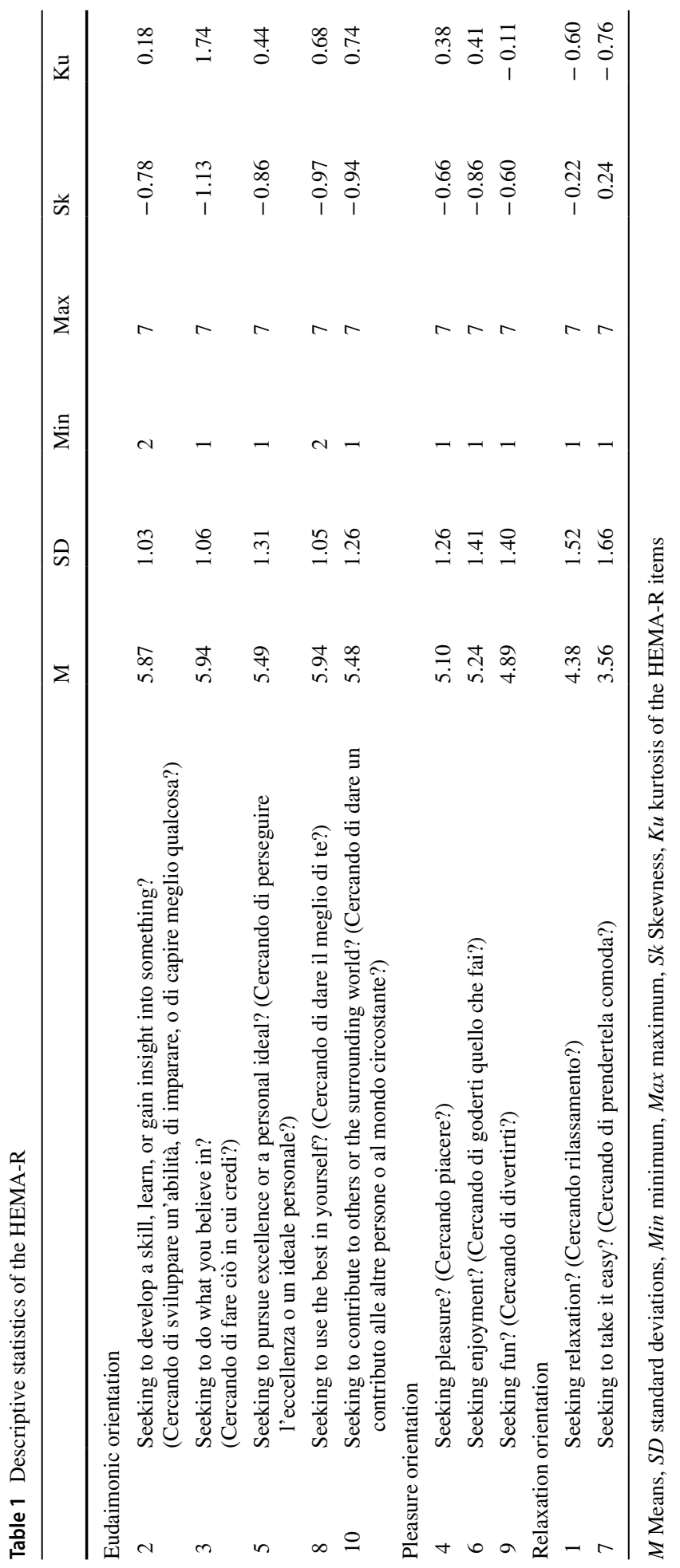




\subsection{Confirmatory Factor Analysis}

The 10 items of the HEMA-R were subjected to a CFA. We specified two models. The two-dimensional model had two correlated factors, namely eudaimonic orientation (items 2, 3, 5, 8 and 10) and hedonic orientation (items 1, 4, 6, 7, and 9). In the second model, we identified three correlated factors with items $2,3,5,8$, and 10 loading on the eudaimonic orientation factor; items 4,6 , and 9 loading on the pleasure orientation factor; and items 1 and 7 loading on the relaxation orientation factor.

The two-dimensional model of the HEMA-R achieved a scarce goodness of fit: $\chi 2(34)=323.5 ; \mathrm{CFI}=0.911 ; \mathrm{TLI}=0.883 ; \mathrm{RMSEA}=0.136(90 \% \mathrm{CI}: 0.123-0.150)$; and $\mathrm{SRMR}=0.102$. By contrast, the three-dimensional model showed adequate fit indices: $\chi 2(32)=115.7 ; \mathrm{CFI}=0.974 ; \mathrm{TLI}=0.964 ; \mathrm{RMSEA}=0.075$ (90\% CI: $0.061-0.090)$; and $\mathrm{SRMR}=0.060$. Hence, we deemed it valid to use the total scores of the eudaimonic orientation, the pleasure orientation and the relaxation orientation subscales for the subsequent analyses. As reported in Fig. 1, factor loadings were all above 0.50. The pleasure factor showed a moderate positive correlation both with eudaimonic $(\phi=0.57)$ and relaxation orientations $(\phi=0.67)$, whereas the correlation between eudaimonic and relaxation orientations was low and negative $(\phi=-0.17)$.

\subsection{Multivariate Linear Model}

We specified a unique multivariate linear model (see Figure S1 in Online Appendix A) in which eudaimonic, pleasure, and relaxation orientations were set as independent variables

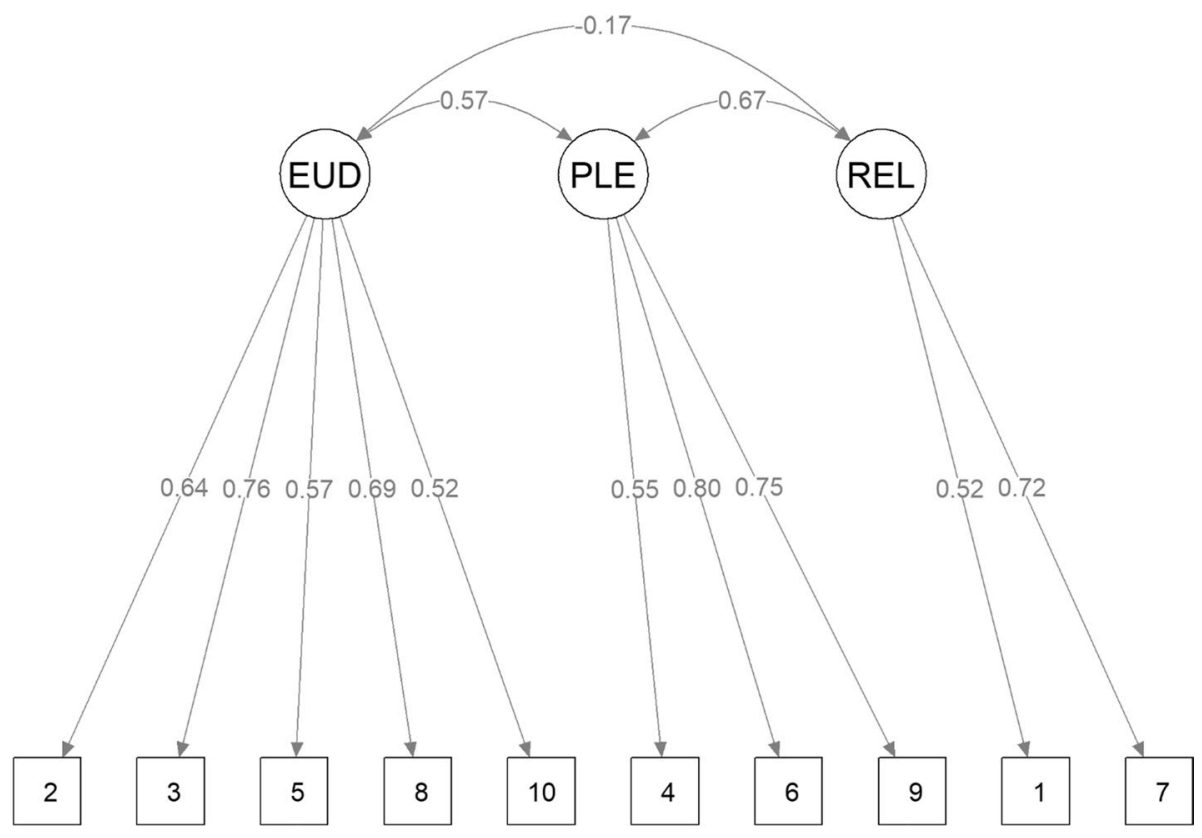

Fig. 1 Confirmatory factor model of the HEMA-R based on a three-factor solution: Eudaimonic orientation (EUD), pleasure orientation (PLE) and relaxation orientation (REL) 
and regressed on well-being positive experiences (flourishing, life satisfaction, and positive affect), negative experiences (negative affect, depression, anxiety, and stress), and functioning measures (i.e., the COPE'S subscales).

Overall, the HEMA dimensions of pleasure, relaxation and eudaimonic orientation explained the $55 \%$ of the variance in the selected outcome measures.

In Table 2, beta coefficients were standardized for direct comparisons. Below, we provide an interpretation of the results focused on a comparison of the effects of the wellbeing orientations on each category of response variables (i.e., well-being positive experiences, negative experiences, and functioning measures).

As regards the positive experiences group of measures, the strongest predictor of positive affect was pleasure orientation, $\beta=0.40, z=8.02, p<0.001,95 \% \mathrm{CI}[0.30,0.50]$, and also for life satisfaction the strongest predictor was pleasure orientation, $\beta=0.36, z=7.14$, $p<0.001,95 \%$ CI $[0.26,0.46]$. Instead, eudaimonic $(\beta=0.30)$ and pleasure $(\beta=0.33)$ orientations showed similar weights for flourishing, 95\% CIs [0.21, 0.38], and [0.24, 0.43], respectively.

For the negative experiences category of response variables, pleasure, among the other types of orientations, was shown to have the strongest, negative, relationship to negative affect, $\beta=-0.32, z=-5.82, p<0.001,95 \%$ CI $[-0.43,-0.21]$, depression, $\beta=-0.39$, $z=-7.42, p<0.001,95 \%$ CI $[-0.49,-0.28]$, anxiety, $\beta=-0.22, z=-3.86, p<0.001$, $95 \%$ CI $[-0.33,-0.11]$, and stress, $\beta=-0.32, z=-5.87, p<0.001,95 \%$ CI $[-0.43$, $-0.21]$.

The relationship between eudaimonic and relaxation orientations on the one hand and negative experiences on the other hand was not significant, except for a weak positive association between eudaimonic orientation and stress. Notably, we found a null zero-order correlation between eudaimonic orientation and stress $(r=-0.03$, see Table $S 1$ in Online Appendix A). Thus, the positive relationship $(\beta=0.12)$ between these two variables in the multivariate linear model can be statistically interpreted as a suppression effect (Horst 1941) of eudaimonic orientation on stress, by virtue of its correlation with pleasure. In other words, acting as a suppressor, eudaimonic orientation purifies the irrelevant variance of stress from pleasure, thus enhancing the relationship between pleasure and stress. However, given that eudaimonic orientation was not directly associated to stress, then the suppression effect can be interpreted as a statistical artefact and not a substantive finding needing explanation.

Thus, whereas both eudaimonic and pleasure orientations were associated with positive experiences, only pleasure orientation showed a negative relationship with negative experiences.

Both adaptive and dysfunctional measures of functioning were listed under the coping strategies dependent variables group. Eudaimonic orientation was associated with several adaptive coping strategies, including active coping, planning, suppression of competing activities, positive reinterpretation and growth, seeking emotional and instrumental social support. Also, eudaimonic orientation was negatively associated with behavioral disengagement. Pleasure orientation was positively associated to humor and positive reinterpretation and growth, whereas it was negatively associated to behavioral disengagement. Finally, relaxation orientation was positively associated with several avoidant coping strategies. Some of these coping strategies do not represent a dysfunctional modality of coping, such as acceptance and humor, but they do not imply active actions to address the situation. Other coping strategies associated with relaxation orientation are instead avoidant in a clear dysfunctional sense, such as mental and behavioral disengagement, denial, restraint, and substance use. 


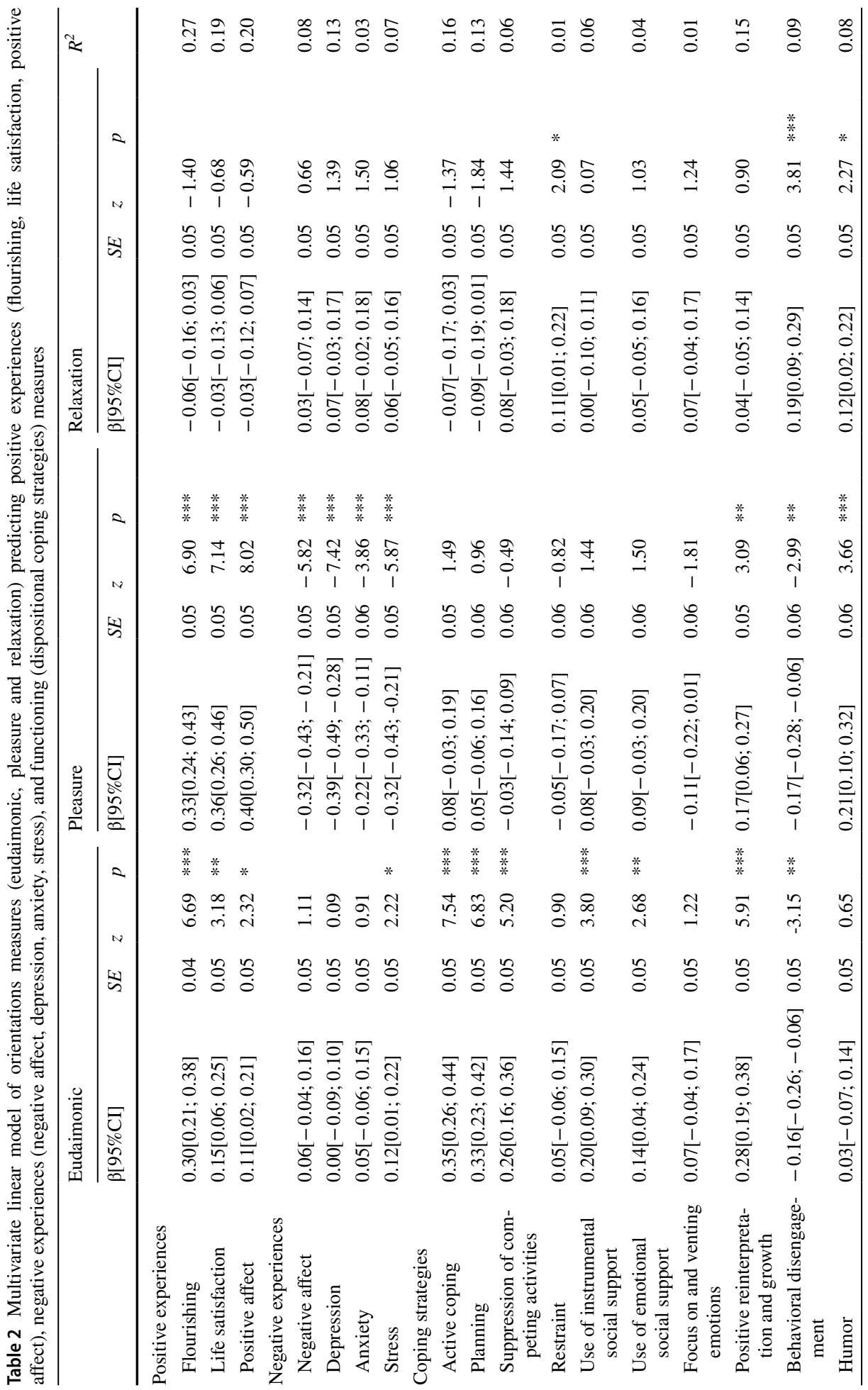




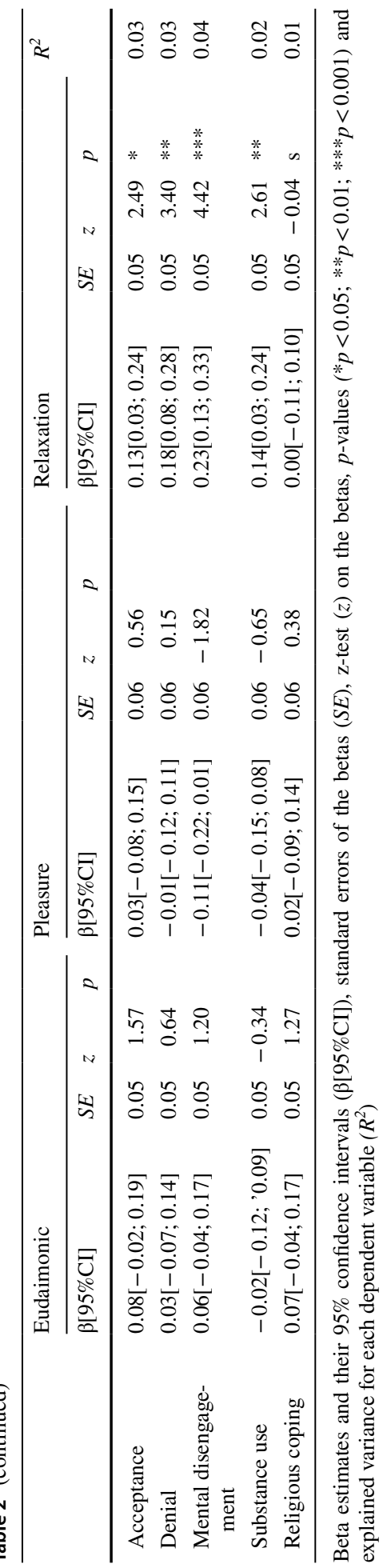




\section{Discussion}

The aim of the current study is based in the idea that the study of wellbeing would benefit from identifying its antecedents, that is, understanding what type of motivations guide individuals in achieving happiness. By using the HEMA-R (Huta and Waterman 2014) in an Italian context, we assessed subjective orientations towards pleasure and comfort (hedonic orientation), and towards self-realization (eudaimonic orientation).

As a preliminary objective, we determined the factorial validity of the Italian version of the HEMA-R by means of a confirmatory factor analysis. Our results supported the three-factor structure of the HEMA-R, providing justification to use the eudaimonic orientation, pleasure orientation, and relaxation orientation subscale scores. This result replicated previous findings obtained with other non-English versions of the HEMA$\mathrm{R}$, in which it was showed that the best fit of the model to the data was obtained by separating the hedonic dimension in orientation to pleasure and orientation to relaxation (Asano et al. 2018; Bujacz et al. 2014).

The main purpose of our research was to explore how different types of orientations to well-being are associated to distinctive types of outcomes measures.

We specified a multivariate linear model to identify the net contributions of eudaimonic, pleasure, and relaxation orientations in explaining different types of outcomes.

Results showed that pleasure orientation was positively associated with positive experiences and negatively associated with negative experiences. In particular, the negative relationship between pleasure orientation and depression was the strongest compared to the other pairs of variables in our model.

Instead, eudaimonic orientation showed lower positive associations than pleasure orientation with positive experiences and no associations with negative experience measures. These findings are consistent with Henderson et al. (2013). Whilst Henderson et al. (2013) interpreted their results as a less relevant role of Eudaimonia than Hedonia in predicting well-being, our findings suggest that Eudaimonia may effectively foster well-being through different pathways.

Indeed, in our study, eudaimonic orientation showed the strongest and the largest number of associations with adaptive approach coping strategies, such as active coping, planning and suppression of competing activities. Although approach coping strategies are not synonymous with immediate well-being and do not imply the absence of emotional distress, they may be conceived as a characteristic of individuals who strive for personal growth on a long-term perspective (Ryff and Singer 2002). Therefore, the positive relationship between eudaimonic orientation and approach coping strategies adds value to the conceptualization of Eudaimonia as a lifelong process in which one develops his or her own core strengths and virtues (Huta and Waterman 2014).

Consistent with previous literature, our results suggest that pursuing both pleasure and Eudaimonia may produce higher degrees of well-being than pursuing only one of the two. According to Huta (2016) Hedonia and Eudaimonia aim to satisfy different types of existential needs, and a full-rounded well-being is achieved when there is a balance between hedonic and eudaimonic orientations. Indeed, our results show that pursuing Eudaimonia is associated with adaptive coping strategies and positive well-being experiences, but the absence of a significant association with negative experiences suggests that an eudaimonic orientation alone does not have a role in diminishing distress. By contrast, hedonic pleasure is directly negatively associated with depression, anxiety, 
and stress, and it is positively associated with adaptive coping strategies, such as humor and positive reinterpretation and growth.

Notably, relaxation taken alone seems to isolate the negative aspect of the hedonic component understood as "pain avoidance". Indeed, on the basis of our results, relaxation was associated with avoidant coping strategies, such as mental and behavioral disengagement, denial, and substance use. Therefore, relaxation orientation may reflect the hedonic part of the avoidance of pain, while pleasure orientation may reflect the hedonic component of the pursuit of pleasure.

As a final remark, it is worth mentioning some of the limitations of this study. First, the cross-sectional design of the study precluded us from providing any causal interpretation of our results. Although we were able to separate the direct effects of the different types of orientation to well-being on several outcome variables, a longitudinal design would be able to test more specific hypotheses. For example, considering coping strategies as mediating variables between orientations and experiences measures, future studies could investigate the long-term effects on hedonic and eudaimonic dimensions of well-being. Second, our study was based on an Italian, high educated, young adult sample, whereas it would be desirable to replicate and extend these findings to different samples and across life-spans. Third, it should also be desirable, instead of measuring flourishing as a one-dimensional construct, to include measures of distinct dimensions of eudaimonic outcomes, such as personal growth, meaning, positive relationships, and engagement. Fourth, in our sample the internal consistency of the COPE's dimensions were sub-optimal for the restraint $(\alpha=0.64)$ and the mental disengagement $(\alpha=0.49)$ subscales, suggesting that results concerning these coping strategies should be regarded with caution. Finally, from a methodological point of view, we deemed it important to control spurious associations between different types of orientations to identify their net contributions in explaining different types of outcomes. However, future research should explore which are the adaptive interactions between eudaimonic, pleasure, and relaxation motives that may help individuals to achieve an optimal functioning in their lives.

In summary, our findings corroborated the good psychometric properties of the HEMA$\mathrm{R}$ through a confirmatory factor analysis of its Italian version. The main contribution of this study was to distinguish pleasure, relaxation, and eudaimonic motives for activities on the basis of their different patterns of correlations with positive and negative experience outcome measures and on the individual's favorite repertoire of coping responses. In particular, compared with eudaimonic orientation, pleasure orientation was associated with higher levels of positive experiences. By contrast, eudaimonic orientation was more typically associated with adaptive coping strategies, as compared to pleasure and relaxation orientations.

Therefore, our paper outlines that future research will need to differentiate between pleasure and relaxation components of hedonic orientation. Furthermore, this study highlights the importance of exploring the relationships between the hedonic (pleasure and comfort) or eudaimonic motives for actions and adaptive coping strategies in order to develop intervention models that could guide individuals in the development of optimal pathways to well-being.

\section{Supplementary Materials}

All relevant data and appendices are available in the Open Science Framework repository, https://osf.io/5m3ty/

Acknowledgements Open access funding provided by Università degli Studi di Padova within the CRUICARE Agreement. 
Data Availability All relevant data and appendices are available in the Open Science Framework repository, https://osf.io/5m3ty/

\section{Compliance with Ethical Standards}

Conflict of interest The authors declare no potential conflicts of interest with respect to the research, authorship, and/or publication of this article.

Ethical Approval All procedures performed in studies involving human participants were in accordance with the ethical standards of the institutional and/or national research committee and with the 1964 Helsinki declaration and its later amendments or comparable ethical standards.

Informed Consent Informed consent was obtained from all individual participants included in the study.

Open Access This article is licensed under a Creative Commons Attribution 4.0 International License, which permits use, sharing, adaptation, distribution and reproduction in any medium or format, as long as you give appropriate credit to the original author(s) and the source, provide a link to the Creative Commons licence, and indicate if changes were made. The images or other third party material in this article are included in the article's Creative Commons licence, unless indicated otherwise in a credit line to the material. If material is not included in the article's Creative Commons licence and your intended use is not permitted by statutory regulation or exceeds the permitted use, you will need to obtain permission directly from the copyright holder. To view a copy of this licence, visit http://creativecommons.org/licenses/by/4.0/.

\section{References}

Anić, P. (2014). Hedonic and eudaimonic motives for favourite leisure activities. Primenjena psihologija, 7(1), 5-21.

Asano, R., Tsukamoto, S., Igarashi, T., \& Huta, V. (2018). Psychometric properties of measures of hedonic and eudaimonic orientations in Japan: The HEMA scale. Current Psychology. https://doi.org/10.1007/ s12144-018-9954-z.

Bottesi, G., Ghisi, M., Altoè, G., Conforti, E., Melli, G., \& Sica, C. (2015). The Italian version of the Depression Anxiety Stress Scales-21: factor structure and psychometric properties on community and clinical samples. Comprehensive psychiatry, 60, 170-181.

Bujacz, A., Vittersø, J., Huta, V., \& Kaczmarek, L. D. (2014). Measuring hedonia and eudaimonia as motives for activities: Cross-national investigation through traditional and Bayesian structural equation modeling. Frontiers in Psychology. https://doi.org/10.3389/fpsyg.2014.00984.

Carver, C. S., Scheier, M. F., \& Weintraub, J. K. (1989). Assessing coping strategies: a theoretically based approach. Journal of personality and social psychology, 56(2), 267-283.

Chua, L. W., Milfont, T. L., \& Jose, P. E. (2015). Coping skills help explain how future-oriented adolescents accrue greater well-being over time. Journal of youth and adolescence, 44(11), 2028-2041.

Deci, E. L., \& Ryan, R. M. (2000). The" what" and" why" of goal pursuits: human needs and the self-determination of behavior. Psychological inquiry, 11(4), 227-268.

Diener, E. D., Emmons, R. A., Larsen, R. J., \& Griffin, S. (1985). The satisfaction with life scale. Journal of personality assessment, 49(1), 71-75.

Diener, E., Wirtz, D., Tov, W., Kim-Prieto, C., Choi, D. W., Oishi, S., et al. (2010). New well-being measures: short scales to assess flourishing and positive and negative feelings. Social Indicators Research, 97(2), 143-156.

Giuntoli, L., Ceccarini, F., Sica, C., \& Caudek, C. (2017). Validation of the italian versions of the flourishing scale and of the scale of positive and negative experience. SAGE Open. https://doi.org/10.1177/21582 44016682293.

Henderson, L. W., Knight, T., \& Richardson, B. (2013). An exploration of the well-being benefits of hedonic and eudaimonic behaviour. The Journal of Positive Psychology, 8(4), 322-336.

Horst, P. (1941). The prediction of personnel adjustment. Social Science Research and Council Bulletin, 48, 431-436. 
Hu, L. T., \& Bentler, P. M. (1998). Fit indices in covariance structure modeling: sensitivity to underparameterized model misspecification. Psychological methods, 3(4), 424.

Huta, V. (2016). Eudaimonic and hedonic orientations: theoretical considerations and research findings. Handbook of eudaimonic well-being (pp. 215-231). Cham, Switzerland: Springer.

Huta, V., \& Ryan, R. M. (2010). Pursuing pleasure or virtue: The differential and overlapping well-being benefits of hedonic and eudaimonic motives. Journal of happiness studies, 11(6), 735-762.

Huta, V., \& Waterman, A. S. (2014). Eudaimonia and its distinction from hedonia: developing a classification and terminology for understanding conceptual and operational definitions. Journal of Happiness Studies, 15(6), 1425-1456.

Kasser, T., \& Ryan, R. M. (2001). Be careful what you wish for: optimal functioning and the relative attainment of intrinsic and extrinsic goals. In P. Schmuck \& K. M. Sheldon (Eds.), Life goals and wellbeing: Towards a positive psychology of human striving (pp. 116-131). Goettingen, Germany: Hogrefe \& Huber.

Keyes, C. L. (2002). The mental health continuum: from languishing to flourishing in life. Journal of health and social behavior, 43(2), 207-222.

Keyes, C. L. (2005). Mental illness and/or mental health? Investigating axioms of the complete state model of health. Journal of consulting and clinical psychology, 73(3), 539-548.

Lovibond, P. F., \& Lovibond, S. H. (1995). The structure of negative emotional states: Comparison of the Depression Anxiety Stress Scales (DASS) with the Beck Depression and Anxiety Inventories. Behaviour research and therapy, 33(3), 335-343.

Moulin, F., Keyes, C., Liu, A., \& Caron, J. (2017). Correlates and predictors of well-being in montreal. Community mental health journal, 53(5), 560-567.

Ortner, C. N., Corno, D., Fung, T. Y., \& Rapinda, K. (2018). The roles of hedonic and eudaimonic motives in emotion regulation. Personality and Individual Differences, 120, 209-212.

Pastore, M. (2012). Modelli di equazioni strutturali per variabili osservate: una nota sugli indici di adattamento. Giornale italiano di psicologia, 39(2), 435-477.

Peterson, C., Park, N., \& Seligman, M. E. (2005). Orientations to happiness and life satisfaction: the full life versus the empty life. Journal of happiness studies, 6(1), 25-41.

R Core Team. (2018). R: A language and environment for statistical computing. Vienna, Austria: R Foundation for Statistical Computing. Retrieved from https://www.Rproject.org/.

Rosseel, Y. (2012). Lavaan: An R package for structural equation modeling and more. Version 0.5-12 (BETA). Journal of statistical software, 48(2), 1-36.

Ryff, C. D. (2014). Psychological well-being revisited: Advances in the science and practice of eudaimonia. Psychotherapy and psychosomatics, 83(1), 10-28.

Ryff, C. D., \& Singer, B. H. (2002). Flourishing under fire: resilience as a prototype of challenged thriving. In C. L. M. Keyes \& J. Haidt (Eds.), Flourishing: Positive psychology and the life well-lived. Washington, D.C.: American Psychological Association.

Seligman, M. E. (2002). Positive psychology, positive prevention, and positive therapy. Handbook of positive psychology, 2(2002), 3-12.

Sica, C., Magni, C., Ghisi, M., Altoè, G., Sighinolfi, C., Chiri, L. R., et al. (2008). Coping Orientation to Problems Experienced-Nuova Versione Italiana (COPE-NVI): uno strumento per la misura degli stili di coping. Psicoterapia cognitiva e comportamentale, 14(1), 27-53.

Sica, C., Novara, C., Dorz, S., \& Sanavio, E. (1997). Coping strategies: Evidence for cross-cultural differences? A preliminary study with the Italian version of coping orientations to problems experienced (COPE). Personality and Individual Differences, 23(6), 1025-1029.

Publisher's Note Springer Nature remains neutral with regard to jurisdictional claims in published maps and institutional affiliations. 